CERN PPE 97-23

MPI-K 97-9

19 February 1997

\title{
$\Xi^{-}$Production by $\Sigma^{-}, \pi^{-}$and Neutrons in the Hyperon Beam Experiment at CERN
}

\section{The WA89 Collaboration}

M.I. Adamovich ${ }^{7}$, Yu.A. Alexandrov ${ }^{7}$, D. Barberis ${ }^{2}$, M. Beck ${ }^{4}$, C. Bérat ${ }^{3}$, W. Beusch ${ }^{1}$, M. Boss ${ }^{5}$, S. Brons ${ }^{4, j}$, W. Brückner ${ }^{4}$, M. Buénerd ${ }^{3}$, C. Busch ${ }^{5}$, C. Büscher ${ }^{4}$, F. Charignon ${ }^{3}$, J. Chauvin ${ }^{3}$, E.A. Chudakov ${ }^{8, h}$, U. Dersch ${ }^{4}$, F. Dropmann ${ }^{4}$, J. Engelfried ${ }^{5, a}$, F. Faller ${ }^{5, b}$, A. Fournier ${ }^{3}$, S.G. Gerassimov ${ }^{4,7}$, M. Godbersen ${ }^{4}$, P. Grafström ${ }^{1}$, Th. Haller ${ }^{4}$, M. Heidrich ${ }^{4}$, E. Hubbard ${ }^{4}$, R.B. Hurst ${ }^{2}$, K. Königsmann ${ }^{4, c}$, I. Konorov ${ }^{4}$, N. Keller ${ }^{5}$, K. Martens ${ }^{5, g}$, Ph. Martin $^{3}$, S. Masciocchi ${ }^{4}$, R. Michaels ${ }^{4, d}$, U. Müller ${ }^{6}$, H. Neeb ${ }^{4}$, D. Newbold ${ }^{4}$, C. Newsom ${ }^{e}$, S. Paul ${ }^{4}$, J. Pochodzalla ${ }^{4}$, I. Potashnikova ${ }^{4}$, B. Povh ${ }^{4}$, Z. Ren ${ }^{4}$, M. Rey-Campagnolle ${ }^{3}$, G. Rosner ${ }^{6}$, L. Rossi $^{2}$, H. Rudolph ${ }^{6}$, C. Scheel ${ }^{h}$, L. Schmitt ${ }^{6}$, H.-W. Siebert ${ }^{5}$, A. Simon ${ }^{5, e}$, V. Smith ${ }^{4}$, O. Thilmann ${ }^{5}$, A. Trombini ${ }^{4}$, E. Vesin ${ }^{3}$, B. Volkemer ${ }^{6}$, K. Vorwalter ${ }^{4}$, Th. Walcher ${ }^{6}$, G. Wälder ${ }^{5}$, R. Werding ${ }^{4}$, E. Wittmann ${ }^{4}$, M.V. Zavertyaev ${ }^{4,7}$

${ }^{1}$ CERN; CH-1211 Genève 23, Switzerland.

${ }^{2}$ Genoa Univ./INFN; Dipt. di Fisica, Via Dodecaneso 33, I-16146 Genova, Italy.

${ }^{3}$ Grenoble ISN; 53 Avenue des Martyrs; CEDEX, F-38026 Grenoble, France.

${ }^{4}$ Heidelberg Max-Planck-Inst. für Kernphysik"; Postfach 103980, D-69029 Heidelberg, Germany.

${ }^{5}$ Heidelberg Univ., Physikal. Inst.\#; Philosophenweg 12, D-69120 Heidelberg, Germany.

${ }^{6}$ Mainz Univ., Inst. für Kernphysik\#; Johann Joachim Becher Weg 45, D-55099 Mainz, Germany.

${ }^{7}$ Moscow Lebedev Physics Inst.; Leninsky Prospect 53, RU-117924, Moscow, Russia.

\section{$\underline{\text { Abstract }}$}

Inclusive cross sections for $\Xi^{-}$hyperon production in high-energy $\Sigma^{-}, \pi^{-}$and neutron induced interactions were measured by the experiment WA89 at CERN. Secondary $\Sigma^{-}$and $\pi^{-}$beams with average momenta of $345 \mathrm{GeV} / c$ and a neutron beam of average momentum $65 \mathrm{GeV} / c$ were produced by primary protons of $450 \mathrm{GeV} / c$ from the CERN SPS. Both single and double differential cross sections are presented as a function of the transverse momentum and the Feynman variable $x_{F}$. A strong leading effect for $\Xi^{-}$produced by $\Sigma^{-}$is observed. The influence of the target mass on the $\Xi^{-}$cross section is explored by comparing reactions on copper and carbon nuclei.

(Submitted to Zeitschrift für Physik C)

\#) supported by the Bundesministerium für Bildung, Wissenschaft,Forschung und Technologie, Germany, under contract numbers 05 5HD15I, 06 HD524I and 06 MZ5265 
${ }^{a}$ Now at FNAL, PO Box 500 Batavia, IL 60510, USA.

${ }^{b}$ Now at Fraunhofer Inst. für Solar Energiesysteme, D-79100 Freiburg, Germany.

${ }^{c}$ Now at Fakultät für Physik, Univ. Freiburg, Germany

${ }^{d}$ Now at Thomas Jefferson Lab 12000 Jefferson Ave., Newport News, VA 23606, USA.

${ }^{e}$ University of Iowa, Iowa City, IA 52242, USA.

${ }^{f}$ Now at Triumf, Vancouver, B.C., Canada V6T $2 A 3$

${ }^{g}$ Now at Kamioka Observatory, Institute for Cosmic Ray Research, University of Tokyo,Japan

${ }^{h}$ On leave from Moscow State University, Moscow, Russia 


\section{Introduction}

The production of strange or heavy quarks and their subsequent hadronization in hadron-hadron collisions constitute an important benchmark test for QCD-inspired phenomenological models [1-5] describing soft phenomena and the applicability of perturbative QCD to hard processes. Using, for example, projectiles with different flavour contents, the role of valence quarks can be explored $[1,3,6]$. In addition, hyperons represent an essential ingredient for our understanding of hadronic systems at high energy density. In reactions with complex nuclei the production of strange $[7,8]$ and charmed [9-15] particles is sensitive to the surrounding nuclear medium. In turn, the produced particles may allow to probe interactions with and properties of the partonic or hadronic medium itself. Evidence for unusual production mechanisms in a non-hadronic environment will always be based on a comparison to hadron-hadron and hadron-nucleus data. Finally, on a macroscopic scale, hyperons are predicted to play a relevant role during the developement of neutron stars [16-18].

The production of hyperons has been studied mostly in proton and neutron induced reactions [19-29] or with heavy ion beams [30-32]. Only few data exist for pions interacting with nuclear targets $[33,34]$. Despite the potential interest, measurements with strange quarks in the incoming beam are also very scarce. $\Xi^{-}$production in $\Xi^{-}+\mathrm{Be}$ and $\Xi^{-}+\mathrm{N}$ interactions was measured in earlier experiments using the charged hyperon beam at CERN [35,36]. Since the various experiments were performed in different kinematic regions, large systematic uncertainties make a direct comparison of these measurements rather difficult.

The latest hyperon beam experiment at CERN - named WA89 - offers the opportunity to measure the $\Xi^{-}$production under homogeneous conditions with different types of beam on several targets. In this paper we present the first measurement of $\Xi^{-}$production in $\Sigma^{-}$interactions, thus filling the gap between non-strange beams and the $\Xi^{-}$induced reactions with two strange quarks in the incoming projectile. In addition, we have studied the $\pi^{-} A \rightarrow \Xi^{-} X$ and $n A \rightarrow \Xi^{-} X$ reactions which enable to connect the new data obtained in the present study with the results of previous measurements.

The paper is organized as follows. Experimental details are presented in the following section 2. In section 3 the results are presented. A comparison with existing data and a discussion follows in section 4 . The final conclusions and a summary are then given in section 5 .

\section{Experimental Setup and Event Selection}

The experiment WA89 was performed using the charged hyperon beam of the CERN SPS. Its main purpose is to study the production, spectroscopy and decays of charmed baryons and to search for exotic states. The production studies of $\Xi^{-}$were performed in parallel with the main program.

\subsection{Beamline and apparatus}

Hyperons were produced by $450 \mathrm{GeV} / c$ protons impinging on a $40 \mathrm{~cm}$ long beryllium target with a diameter of $0.2 \mathrm{~cm}$. A magnetic channel consisting of 3 magnets with an integrated field of 8.4 Tm selected negative particles with a mean momentum of $345 \mathrm{GeV} / c$, a momentum spread of $\sigma(p) / p=9 \%$, and an angle to the proton beam smaller than $0.5 \mathrm{mrad}$. After a distance of $16 \mathrm{~m}$ the produced hyperons hit the experimental target which consisted of one copper and three carbon (diamond) blocks arranged in a row along the beam. Each copper and carbon block had a thickness corresponding to an interaction length of $2.6 \%$ and $0.83 \%$, respctively. At the target the beam was homogeneously distributed over a rectangular area with a width of $3 \mathrm{~cm}$ and a height of $1.7 \mathrm{~cm}$. Its dispersion was $0.6 \mathrm{mrad}$ in the horizontal plane and $1.0 \mathrm{mrad}$ in the vertical plane. 
An average beam spill of $2.1 \mathrm{~s}$ contained about $1.8 \cdot 10^{5} \Sigma^{-}$hyperons and about $4.5 \cdot 10^{5} \pi^{-}$at the experimental target for an incoming intensity of $4.0 \cdot 10^{10}$ protons per spill. A transition radiation detector (TRD) [37] was used to discriminate online between $\pi^{-}$and hyperons. In special runs $\pi^{-}$ interactions were recorded for normalisation purposes.

Fig. 1 shows a sketch of the experimental setup used in the 1993 run of WA89 on which the data of this paper are based. The beam and the secondary particles were detected by 29 silicon micro-strip planes with 25 and $50 \mu \mathrm{m}$ pitch. Positioning the target about $14 \mathrm{~m}$ upstream of the centre of the $\Omega$-spectrometer provided a $10 \mathrm{~m}$ long decay area for short living strange particles. The products of these decays along with the particles coming directly from the target were detected by 40 planes of drift chambers with a spatial resolution of about $300 \mu \mathrm{m}$. Special MWPC chambers $(20$ planes with $1 \mathrm{~mm}$ wire spacing) were used in the central region of high particle fluxes. In order to improve the track bridging between the target region and the decay region three sets of 4 MWPCs each with a pitch of $1 \mathrm{~mm}$ were installed about $2 \mathrm{~m}$ behind the target.

The particle momenta were measured by the $\Omega$-spectrometer [38] consisting of a super-conducting magnet with a field integral of $7.5 \mathrm{Tm}$ and a tracking detector consisting of $45 \mathrm{MWPC}$ planes inside the field area and 12 drift chamber planes at the exit of the magnet. The momentum resolution was $\sigma(p) / p^{2} \approx 10^{-4}(\mathrm{GeV} / c)^{-1}$.

Charged particles were identified using a ring imaging Cherenkov (RICH) detector [39]. It had a threshold of $\gamma=42$ and provided $\pi / p$ separation up to about $150 \mathrm{GeV} / c$. Downstream of the RICH a lead glass electromagnetic calorimeter was positioned for photon and electron detection [40]. This calorimeter was followed by a hadron calorimeter [41].

The trigger selected about $25 \%$ of all interactions using multiplicities measured in scintillators in the target region and in hodoscopes and proportional chambers downstream of the magnet. Correlations of hits in these detectors were used to select particles with high momenta thus reducing the background from low-momentum pions (see section 2.3.1). More than 2 particles at the exit of the magnet were required by the trigger. The results shown in the present paper are based on the analysis of about 100 million events recorded in 1993.

\subsection{Event reconstruction}

The event reconstruction proceeded in two steps. In the first part of the analysis events with a $\Xi^{-}$candidate were selected. Secondly, interactions in the target were identified, with different constraints applied for the different beam particles.

\subsection{1 $\Xi^{-}$identification}

$\Xi^{-}$were reconstructed in the decay chain $\Xi^{-} \rightarrow \Lambda^{0} \pi^{-} \rightarrow p \pi^{-} \pi^{-}$. Only $\Lambda^{0}$-decays upstream of the magnetic field of the $\Omega$-spectrometer were considered in this analysis. Reconstructed segments in the chambers of the decay area and in the $\Omega$-spectrometer were required for the tracks of the daughter particles

Candidates for $\Xi^{-}$-decays were then selected by the following procedure. For the definition of the $\Lambda^{0} \rightarrow p \pi^{-}$decays, all combinations of positive and negative tracks were considered. The distance of the two tracks at the decay point was not allowed to exceed $0.5 \mathrm{~cm}$. To look for $\Xi^{-} \rightarrow \Lambda^{0} \pi^{-}$decays, only $\Lambda^{0}$ candidates within a mass window of $3 \sigma$ around the reference mass were accepted. Here $\sigma$ denotes the uncertainty of the mass determination based on the track properties of the individual events. (Typically, $\sigma$ is about $3.7 \mathrm{MeV} / c^{2}$ ). In addition, the corresponding $\Xi^{-}$ trajectory had to be measured in the vertex detector. The momentum spectrum of the $\Xi^{-}$and $\Lambda^{0}$ candidates starts at about $12 \mathrm{GeV} / c$, the cutoff being due to the spectrometer acceptance. 


\subsubsection{Selection of interactions}

$\Sigma^{-}, \pi^{-}$and neutron interactions were identified according to the following conditions. The interaction vertex contains at least two outgoing charged tracks one of which is the $\Xi^{-}$track. Furthermore, the reconstructed vertex position had to be within a target block where in each coordinate an additional margin of $3 \sigma$ was allowed. The $\sigma$ denotes the uncertainty of the vertex position calculated for each individual event from the track parameters.

For $\Sigma^{-}$and $\pi^{-}$interactions the transverse distance between the $\Sigma^{-}$or $\pi^{-}$beam track and the reconstructed interaction vertex position was required to be less than $6 \sigma(\sigma \approx 25 \mu \mathrm{m})$. Events were rejected if the beam track was connected to an outgoing track.

To identify interactions of neutrons generated by $\Sigma^{-}$decays in the beam channel the following criteria were imposed. Since $\pi^{-}$from $\Sigma^{-}$decays are below the TRD threshold, it was required that no high momentum $\pi^{-}$was detected in the TRD. In addition, the $\pi^{-}$track from the $\Sigma^{-}$decay had to pass the reconstructed interaction point with a distance of at least $6 \sigma$. Finally, it was demanded that the $\pi^{-}$track was connected to a track in the spectrometer and that it had a momentum smaller than $140 \mathrm{GeV} / c$ corresponding to the $\Sigma^{-} \rightarrow n \pi^{-}$decay kinematics at $\langle p>\approx 345 \mathrm{GeV} / c$.

The resulting $\Xi^{-}$mass spectra for the different incident beam particles are shown in Fig. 2 . We observed about $178,000 \Xi^{-}$from $\Sigma^{-}$interactions, $3000 \Xi^{-}$from $\pi^{-}$interactions and 3500 from neutron interactions. The mass resolution lies in the range from $2.5 \mathrm{MeV} / c^{2}$ to $2.8 \mathrm{MeV} / c^{2}$ and is dominated by the error on the direction of the decay products.

\subsection{Beam properties}

The negative beam particles ( $\Sigma^{-}, \pi^{-}$) had an average momentum of $345 \mathrm{GeV} / c$ and a momentum spread of $\sigma(p) / p=9 \%$. Since the beam momentum in each individual event was not measured, we used an average momentum of $345 \mathrm{GeV} / c$ for the further analysis.

Despite the beam particle identification in the TRD, the $\Sigma^{-}$or $\pi^{-}$data taken in the present experiment were contaminated by misidentified $\pi^{-}, \Sigma^{-}, \Xi^{-}, \mathrm{K}^{-}$and neutrons. For all our measurements the number of $\Xi^{-}$in the beam is most crucial due to the large $\Xi^{-}$production cross section in the reaction $\Xi^{-}+A \rightarrow \Xi^{-}+X$ [35]. Therefore the beam composition and its influence on the cross section measurement was studied in a detailed analysis for each data set individually.

\subsubsection{The $\Sigma^{-}$beam}

To measure the $\Xi^{-}$contamination of the $\Sigma^{-}$beam, a sample of events with beam particles passing through the target without interaction was analyzed. In this data sample we identified $\Sigma^{-}$and $\Xi^{-}$ decays by the observation of the decay kinks between the incoming beam particle and a negative particle detected in the spectrometer from the decays $\Sigma^{-} \rightarrow n \pi^{-}$and $\Xi^{-} \rightarrow \Lambda \pi^{-}$, respectively. The decay point was accepted if it was located between the last micro strip plane and the first set of drift chambers of the decay area. The decay lengths of $\Sigma^{-}$and $\Xi^{-}$at the same momentum differ by only $1 \%$ and therefore no acceptance or efficiency corrections are needed to evaluate the $\Sigma^{-} / \Xi^{-}$ ratio. In part a) of Fig. 3 the expected correlation between the momentum and the kink angle is shown for $\Sigma^{-}$and $\Xi^{-}$decays. In order to determine the separation between the different beam particles, an equal number of $\Sigma^{-}$and $\Xi^{-}$particles was generated in this Monte Carlo simulation. The experimental correlation between the momentum and the kink angle is displayed in Fig. $3 \mathrm{~b}$. A second band originating from $\Xi^{-}$decays can be discerned there. For a quantitative analysis we projected this distribution along the angle-momentum correlation marked by the dashed and solid lines. This projection is shown by the solid histogram in part c) of Fig. 3. Besides the dominating peak from $\Sigma^{-}$decays, a well separated maximum corresponding to $\Xi^{-}$decays can be identified. The 
relative strength of the two peaks can be estimated with the Monte Carlo simulations by assuming a $\Xi^{-}$contribution of $(1.26 \pm 0.07) \%$ to the $\Sigma^{-}$beam (see cross-hatched histogram in Fig. 3.c). Based on the $\Xi^{-}+B e \rightarrow \Xi^{-}+X$ cross section from Ref. [36] the differential cross sections were corrected for this contamination.

The actual $\pi^{-}$to $\Sigma^{-}$ratio in the beam is about 2.3. Although beam $\Sigma^{-}$were identified online by the TRD, the data sample taken during the experiment was still contaminated by misidentified $\pi^{-}$with large momenta. The amount of this contamination was determined offline with the help of the pulse height information from each chamber in the TRD. A value of $(12.3 \pm 0.5) \%$ of remaining high momentum $\pi^{-}$in the $\Sigma^{-}$data sample was obtained. Using the $\pi^{-}+A \rightarrow \Xi^{-}+X$ cross section measured in the present experiment (see below), we corrected the observed $\Sigma^{-}+A \rightarrow \Xi^{-}+X$ yield in an iterative way for these $\pi^{-}$interactions.

The $K^{-}$to $\pi^{-}$ratio in $300 \mathrm{GeV} / c$ p-Be collisions was measured to be $(1.15 \pm 0.02) \%$ [42]. Since high momentum $K^{-}$mesons cannot be separated from the $\Sigma^{-}$beam via the TRD information, $2.1 \%$ of the total beam in our data sample consists of $K^{-}$. The production cross section for $K^{-} p \rightarrow \Xi^{-} X$ was measured only at low beam momenta between 4.2 and $16 \mathrm{GeV} / c$ [24]. Within this momentum range the cross section slowly decreases from $157 \pm 8 \mu b$ to $135 \pm 15 \mu b$. Taking $157 \mu b$ as an upper limit for the cross section of $\Xi^{-}$produced by $K^{-}$at $345 \mathrm{GeV} / c$ we estimated that at most $0.4 \%$ of the observed $\Xi^{-}$yield can be attributed to $K^{-}$content of the beam. Since the actual value for the $K^{-}$cross section is unknown we include this contamination in the systematic error.

Finally, the $\Sigma^{-}$data sample was contaminated by low momentum pions and neutrons stemming from $\Sigma^{-}$decays between the exit of the beam channel and the target. Monte Carlo simulations predicted that $28 \%$ of the $\Sigma^{-}$at the exit of the beam channel decayed with the daughter $\pi^{-}$hitting the beam scintillators and the target. Thus, slow secondary pions should acount for about $24 \%$ of the total flux at the target. According to these simulations a strong interdependence between the incident angle and the position at the target is expected for the primary beam particles while no such correlation exists for slow, secondary pions. Indeed a plot for the experimental data of the beam angle versus the target position shows a narrow band which is superimposed on a homogeneous background. We find that $23 \%$ of the total incoming beam flux is contained in this background. Motivated by the good agreement of this number with the expected $24 \%$ of secondary pions, we attribute the particles outside of the narrow position-angle correlation band to decay pions. We therefore corrected the total incident beam flux for a $23 \%$ contribution from low momentum pions. By cutting on the position-angle correlation the contamination to the observed $\Xi^{-}$yield from these slow pions could be suppressed.

\subsubsection{The $\pi^{-}$beam}

To obtain a sample of interactions of beam pions the online TRD decision was inverted. This data sample contains misidentified $\Sigma^{-}, \Xi^{-}$, and $K^{-}$-mesons. With the help of the TRD offline analysis we determined the total remaining contamination in the pion beam to be $(1.5 \pm 1) \%$. In order to correct the pion data for this background, we assumed that the relative distribution between $\Sigma^{-}, \Xi^{-}$and $\mathrm{K}^{-}$is identical to that of the original beam of about $78: 1: 3$. Using the $\Sigma^{-}+A \rightarrow \Xi^{-}+X$ cross section from the present study and the $\Xi^{-}+B e \rightarrow \Xi^{-}+X$ data at 116 $\mathrm{GeV} / c$ [36], we obtained corrections of about $10 \%$ and $1.5 \%$ due to the $\Sigma^{-}$and $\Xi^{-}$contributions, respectively. Because of the additional TRD suppression, the contribution to the $\pi^{-}$sample from the $K^{-}$component is negligible. 


\subsubsection{The neutron beam}

Neutrons originating from $\Sigma^{-}$decays upstream of the target were used to measure the $\Xi^{-}$production by neutrons. The momenta of these neutrons were defined as the difference between the average $\Sigma^{-}$momentum and the momentum of the associated $\pi^{-}$measured in the spectrometer. The neutron spectrum has an average momentum of $260 \mathrm{GeV} / c$ and a width of $\sigma(p) / p=15 \%$.

Despite the tagging of the neutron interactions by the associated pions, this data sample is also not free of contaminations. $\Lambda^{0}$ stemming from $\Xi^{-}$decays in front of the target show a similar event topology. However, due to the small $\Xi^{-}$content of the beam (see section 2.3.1) and the decay of $\Lambda^{0}$ in flight upstream of the target, the content of $\Lambda^{0}$ in the neutron beam amounts to less than $1.26 \%$. On the basis of quark counting rules, we do not expect that the $\Lambda^{0}(u d s)+A \rightarrow \Xi^{-}(d s s)+X$ cross section exceeds the one of the $\Sigma^{-}(d d s)+A \rightarrow \Xi^{-}(d s s)+X$ reaction. Based on the cross section measured in the present experiment, we find that in the $x_{F}$ region of interest $\left(x_{F} \leq 0.6\right)$ corrections due to $\Lambda^{0}$ interactions reach at most $22 \%$ at large $x_{F}$. For small $x_{F} \approx 0$ this contamination falls below $2 \%$. Since the $\Lambda^{0}$ cross section is not known, we attribute these potential corrections to the systematic uncertainties.

We also estimated the probability for events with two beam particles, where one particle was not reconstructed and thus giving the same topology as a neutron interaction. We therefore scanned our data for events with a beam $\Sigma^{-}$reconstructed in the hadron calorimeter and an interaction vertex without a beam track pointing to it. The contamination of the neutron data sample from such type of events was found to be negligible.

\subsection{Detection Efficiencies}

The large aperture of the spectrometer provided a relatively flat geometrical acceptance within the region of $x_{F} \geq 0.05$ and $p_{T} \leq 2.5 \mathrm{GeV} / c$. However, detailed Monte Carlo calculations are required in order to determine the reconstruction efficiency for the decaying hyperons. The primary $\Xi^{-}$ momentum distribution was generated following a kinematical distribution of the form

$$
\frac{d^{2} \sigma}{d p_{t}^{2} d x_{F}}=C\left(1-x_{F}\right)^{n} \cdot \exp \left(-b p_{t}^{2}\right)
$$

For each generated $\Xi^{-}$, the remaining momentum was assigned to a virtual $\pi^{-}$which was fed as a beam particle into the FRITIOF simulation package [43] to generate the correlated hadronic background. A complete detector simulation was performed in the frame of GEANT [44]. These simulations took all available information on the detection efficiencies of the individual components of the tracking system into account. The simulated events were subsequently passed through the event reconstruction chain described above. To check if the Monte Carlo corrections are self consistent, we produced two independent sets of events with different $x_{F}$ and $p_{t}^{2}$ parametrization. We were able to restore the generated $x_{F}$ and $p_{t}^{2}$ distributions of the first set applying the corrections obtained in the second set.

The overall detection efficiency for $\Xi^{-}$decays reaches a maximum of about $6 \%$ at $x_{F}=0.25$. Towards low $x_{F} \approx 0$ the efficiency decreases to about $2.5 \%$ mainly due to acceptance losses for large-angle tracks and to the smaller reconstruction efficiency for low momentum tracks. At high $x_{F} \approx 1$ the efficiency drops to $1.1 \%$ because of the finite fiducial volume for $\Xi^{-} \Lambda^{0}$ decays. According to the simulation, no significant difference in the detection efficiency is expected for $\Xi^{-}$produced in the carbon and copper targets.

The uncertainty in the efficiency determination is the major source of systematic error in the cross section measurements. To estimate this uncertainty we varied the event selection criteria 
applied to the data and the simulated events. The resultant changes in the cross sections are about $20 \%$.

Finally, uncertainties of the incident beam flux were estimated by subdividing the data into subsamples and normalizing them individually. We found maximum variations of $15 \%$.

Adding all systematic errors quadratically we derive at a total systematic uncertainty of $25 \%$ for the $\pi^{-}$and $\Sigma^{-}$measurements. For the neutron beam, the systematic uncertainty rises with $x_{F}$ from $26 \%$ at $x_{F}=0$ to about $33 \%$ at $x_{F}=1$.

\section{Results}

\subsection{Differential cross sections}

The number of observed $\Xi^{-}$decays was translated into a cross section using the formula:

$$
\sigma\left(x_{F}, p_{t}^{2}\right)=\frac{1}{B R\left(\Lambda^{0} \rightarrow p \pi^{-}\right)} \cdot \frac{N_{\Xi^{-}}}{\varepsilon\left(x_{F}, p_{t}^{2}\right) N_{b} \rho l N_{A} / M} .
$$

Here $N_{\Xi^{-}}$is the number of observed $\Xi^{-}, \varepsilon$ denotes the efficiency (including the trigger efficiency) and acceptance, $N_{b}$ is the number of incoming beam particles corrected for the corresponding beam contaminations and losses due to the dead time of the trigger and the data acquisition system. The target has an atomic mass $M$, a density $\rho$, and a geometric length $l . N_{A}$ stands for the Avogadro number and $B R\left(\Lambda^{0} \rightarrow p \pi^{-}\right)$accounts for the branching ratio of the observed $\Lambda^{0}$ decay mode.

Figures 4 and 5 display the differential cross sections as a function of the Feynman variable, $x_{F}$, and the squared transverse momentum, $p_{t}^{2}$, for the three different projectiles and the copper and the carbon targets, respectively. Only statistical errors are shown. The corresponding numbers are listed in tables 1 - 2 .

As a point of reference, we parametrized the observed invariant cross sections by a function of the form

$$
\frac{d^{2} \sigma}{d p_{t}^{2} d x_{F}}=C\left(1-x_{F}\right)^{n} \cdot \exp \left(-b p_{t}^{2}\right)
$$

which is based on quark counting rules [45] and phase space arguments. The three parameters C, $\mathrm{b}$, and $\mathrm{n}$ were assumed to be independent of $p_{t}$ and $x_{F}$. In case of $\Sigma^{-}$interactions this functional form parametrizes the data only in the regions of $x_{F}>0.4$ and $p_{t}^{2}<1 \mathrm{GeV}^{2} / c^{2}$. Fits within this limited range are shown by the solid lines in Figs. 4 and 5 . The values of the fit parameters are listed in Table 3. To obtain the total production cross section we integrated the differential cross sections in the region $0<x_{F}<1$. For neutron and $\pi^{-}$interactions the fits shown in Figs. 4 and 5 were used to extrapolate from the measured range up to $x_{F}=1$. This cross section is also listed in Table 3 .

The distributions shown in Figure 4 signal a strong leading effect for the $\Sigma^{-}$projectile at large $x_{F}$. In this projectile fragmentation region the quark overlap between projectile and produced particle is reflected in the production yield. In turn, in the central region the different beam particles yield comparable $\Xi^{-}$cross sections thus indicating that the initial strangeness content in the projectile is not relevant and all strange quarks are produced in the fragmentation process.

The transverse momentum distributions are - for $p_{t} \leq 1 \mathrm{GeV} / c-$ similar for the different projectiles. The most striking feature in Figure 5 is a strong enhancement at large transverse momenta for the $\Sigma^{-}$data as compared to the $\mathrm{n}$ and $\pi^{-}$data. The slope of this high momentum tail is about half of the one describing the low $p_{t}$ regime, a phenomenon which is consistent with early observations at the CERN-ISR [46,47]. It is an indication that at $p_{t}^{2} \approx 1 \mathrm{GeV}^{2} / c^{2}$ higher order double scattering processes become important. 
For the high statistics $\Sigma^{-}$data we present the invariant double differential cross sections in Tables $4-5$ corrected for the different components of our beam. The error bars contain the statistical error contribution from data and Monte Carlo.

\section{$3.2 \quad A$ dependence}

Acceptance and efficiency corrections for the detected $\Xi^{-}$yield do not depend on the target. Therefore the ratio of the cross sections for the copper and carbon targets allows the determination of the dependence of $\Xi^{-}$production on the nuclear mass $A$ with negligible systematic uncertainties. Figure 6 shows the variation of the normalized cross section ratio

$$
R=\frac{\sigma_{C u}}{\sigma_{C}} \cdot \frac{A_{C}}{A_{C u}}
$$

as a function of $x_{F}$ (top) and $p_{t}^{2}$ (bottom). Here, the indices $\mathrm{C}$ and $\mathrm{Cu}$ refer to the carbon and copper target, respectively. Using the conventional parametriszation for the $A$ dependence

$$
\sigma=\sigma_{0} \cdot A^{\alpha}
$$

the ratio $R$ can be directly translated into the exponent $\alpha$ (righthand scale in Fig. 6 ). The average values of this attenuation factor $\alpha$ together with the extrapolated inclusive cross section per nucleon are listed in Table 6.

A compilation of particle production yields in proton - nucleus collisions given in ref. [48] suggested for $x_{F}>0$ a dependence of $\alpha$ on $x_{F}$ of the form $\alpha\left(x_{F}\right)=0.8-0.75 x_{F}+0.45 x_{F}^{2}$. While the attenuation for $\Xi^{-}$production in $\Sigma^{-}$induced interactions is well described by this parametrization (solid line in the upper part of Figure 6), the neutron and pion data show significantly larger values for $\alpha$ at low $x_{F}$. This deviation from the general behaviour is in line with the target mass dependence observed earlier for $\Xi^{0}$ production in proton induced reactions at $400 \mathrm{GeV} / c[27,48,49]$. It seems that the production of two additional strange quarks at small $x_{F}$ is generally related to a weaker attenuation. Within the constituent quark model such a behaviour can be explained by the assumption that a recombination of leading quarks with a heavy strange quark is suppressed compared to the recombination with a light up or down quark [1].

\section{Comparison with other data}

In the left part of figure 7 we compare the invariant cross section per nucleon measured in neutron induced reactions with data from proton induced reactions at similar energies. As naively expected from the larger d-quark content in the incoming neutron, the neutron data lie slightly above the cross section for $p A$ collisions.

The invariant cross section for $\Xi^{0}$ production in p-nucleon collisions is given by the stars in Fig. 7 (left). Here, the p-Be data from ref. [27] were extrapolated to a nucleon target via a power-law $A$ dependence of the form $A^{0.8}$. Assuming isospin symmetry, identical cross sections are expected for the $\mathrm{n}(u d d)+A \rightarrow \Xi^{-}(\mathrm{dss})$ and the $\mathrm{p}(u u d)+A \rightarrow \Xi^{0}(u s s)$ reactions. However, the present $n A \rightarrow$ $\Xi^{-} \mathrm{X}$ cross section is about a factor of 5 above the $\mathrm{p} A \rightarrow \Xi^{0} \mathrm{X}$ data from ref. [27]. At present, no explanation for this unexpected inconsistency exists.

For pion induced $\Xi^{-}$production we observe a cross section which is about a factor 2 higher than those measured previously in $\pi+A$ collisions at $200 \mathrm{GeV} / c$ [34] (right part of figure 7). In contrast to this old measurement the present values are only about $50 \%$ smaller than the corresponding cross section for proton induced reactions. This difference is close to the ratio of the total inelastic 
cross sections for $\pi^{-}$and protons. Finally, we note that our value for the exponent $\mathrm{n}$ describing the $x_{F}$ dependence is only about half of that observed earlier for $\Xi^{ \pm}$production $(\mathrm{n}=6.7 \pm 0.3)$ in ref. [33].

To check our possible systematic errors we performed a cross section measurement for $\Lambda^{0}$, $\bar{\Lambda}^{0}$ and $K^{0}$ in pion and neutron interactions. These cross section values will be the subject of a forthcoming paper. They are in agreement with the existing world data within $40 \%$ of its values and show us that there we don't have additional unknown systematic errors big enough to explain the observed difference between the $\mathrm{p} A \rightarrow \Xi^{0}$ and $\mathrm{p} A \rightarrow \Xi^{-}$production cross sections.

In fig. 8 we compare our data on $\Xi^{-}$production by $\Sigma^{-}$with other production cross sections of hyperons by different projectiles. Figure 8 (left part) shows the $x_{F}$ dependence of $\Xi^{-}$production by protons, $\Sigma^{-}$and $\Xi^{-}$. Although projectile and produced $\Xi^{-}$differ in strangeness by different amounts the three cross sections are equal at small $x_{F}$. At large $x_{F}$ strong leading effects are observed for $\Xi^{-}$as well as $\Sigma^{-}$projectiles. At $x_{F}$ close to unity, each unit of strangeness in the incoming projectile causes a cross-section increase by about two orders of magnitude.

A complementary behaviour is observed in the right part of Figure 8 which compares three reactions, where the incoming projectile and the produced particle differ by one unit of strangeness. Here the cross sections are of approximatly equal size at large $x_{F}$, but differ by about one order of magnitude at small $x_{F}$. Together the two figures indicate that in the central region the initial strangeness content in the projectile is not relevant for the production of $\Xi^{-}$and all strange quarks contained in the $\Xi^{-}$are produced in the fragmentation process. On the other hand, in the projectile fragmentation region the cross sections depend strongly on the overlap of the quark content between the projectile and the produced particle.

\section{$5 \quad$ Summary and conclusions}

We have presented the first measurement of $\Xi^{-}$production by $\Sigma^{-}$, at a beam energy of $345 \mathrm{GeV} / \mathrm{c}$ and using carbon and copper targets. The measurement was supplemented by measurements of $\Xi^{-}$ production by neutrons and $\pi^{-}$, with lower statistics.

At $p_{t}$ values above $1 \mathrm{GeV} / \mathrm{c}$, the cross-section for $\Xi^{-}$production by $\Sigma^{-}$shows a marked increase compared to the usually observed Gaussian behaviour of $d \sigma / d p_{t}^{2}$, which indicates that a different production process becomes important.

The A-dependence of the $\Xi^{-}$production cross-section is close to a value of $\alpha=2 / 3$ for production by $\Sigma^{-}$, while the less precise data on production by neutrons and $\pi^{-}$favour a higher value $\alpha=0.9$. All three cross-sections show a trend to decreasing values of $\alpha$ with increasing $x_{f}$.

Difficulties encountered in comparing absolute cross-sections from different experiments with pion and neutron beams underline the necessity of comprehensive high-statistics studies of particle production within one experiment for more detailed investigations of hadronic interactions.

Nonetheless, a comparison of our result on the differential cross-section $d \sigma / d x_{F}$ for $\Xi^{-}$production by $\Sigma^{-}$with existing data on hyperon production by nucleons and $\Xi^{-}$shows a strong leading particle effect: at low $x_{F}$ the baryon production cross-sections depend strongly on the strangeness of the produced particle, and are independent of the quark flavour of the beam particle. At high $x_{F}$, on the other hand, the cross-sections depend strongly on the strange quark overlap between beam particle and produced particle, i.e. the cross-sections at high $x_{F}$ are strongly enhanced by quark transfer from the beam particle to the outgoing particle. These results emphasize the power of experiments with beams of different strangeness to distinguish between different contributions to the complex phenomenon of inclusive hadronic production. 


\section{Acknowledgements}

We are indebted to J.Zimmer and the late Z.Kenesei for their help during all moments of detector construction and set-up. We are grateful to the staff of CERN's EBS group for providing an excellent hyperon beam channel, to the staff of CERN's Omega group for their help in running the $\Omega$-spectrometer and also to the staff of the SPS for providing good beam conditions. We thank D.M.Jansen, B.Kopeliovich, R.Ransome and O.Piskounova for many helpful discussions. 


\section{References}

[1] F. Takagi, Phys. Rev. D 27, 1461 (1983).

[2] T. Tashiro et al., Z. Phys. C - Particles and Fields 35, 21 (1987).

[3] T. Tashiro, H. Noda, and K. Kinoshita, Z. Phys. C - Particles and Fields 39, 499 (1988).

[4] R.A.M.S. Nazareth, N. Prado, and T. Kodama, Phys. Rev. D 40, 2861 (1989).

[5] R.A.M.S. Nazareth, T. Kodama, and D.A. Portes, Phys. Rev D 46, 2896 (1992).

[6] F.O. Durães et al., Phys. Rev. D 53, 6136 (1996).

[7] J. Rafelski and B. Müller, Phys. Rev. Lett. 48, 1066 (1982);

Phys. Rev. Lett. 56, 2334 (1986) (E).

[8] P. Koch, B. Müller, and J. Rafelski, Phys. Rep. 142, 167 (1986).

[9] T. Matsui and H. Satz, Phys. Lett. B178, 416 (1986).

[10] K. Geiger, Phys. Rev. D 48, 4129 (1993).

[11] R. Vogt, B.V. Jacak, P.L. McGaughey, P.V. Ruuskanen, Phys. Rev. D 49, 3345 (1994).

[12] P. Levai, B. Müller, and Xin-Nian Wang, Phys. Rev. C 51, 3326 (1995).

[13] Z. Lin and M. Gyulassy, Phys. Rev. C 51, 2177 (1995); Phys. Rev. C 52, 440 (1995) (E).

[14] E. Shuryak, nucl-th/9605011 (1996).

[15] R.C. Hwa, Phys. Rev. Lett. 77, 227 (1996).

[16] N.K. Glendenning, Phys. Lett. 114B, 392 (1982).

[17] N.K. Glendenning, Z. Phys. A 326, 57 (1987).

[18] J. Schaffner and I.N. Mishustin, Phys. Rev. C 53, 1416 (1996).

[19] J. Badier et al., Phys. Lett. B39, 414 (1972).

[20] V. Hungerbühler et al., Phys. Rev. D 12, 1203 (1975).

[21] P. Skubic et al., Phys. Rev D 18, 3115 (1978).

[22] M. Bourquin et al., Nucl. Phys. B 153, 13 (1979).

[23] S. Erhan et al., Phys. Lett. B 85, 447 (1979).

[24] M. Baubillier et al., Nucl. Phys. B 192, 1 (1981).

[25] M. Bourquin et al., Z. Phys. C 5, 275 (1980).

[26] T.R. Cardello et al., Phys. Rev. D 32, 1 (1985).

[27] A. Beretvas et al., Phys. Rev. D 34, 53 (1986).

[28] E.E. Gottschalk et al., Phys. Rev. D 53, 4756 (1996).

[29] A.N.Aleev et al., Yad. Fiz. 44, 661 (1986)

[30] S. Abatzis et al., Phys. Lett. B 259, 508 (1991).

[31] E. Andersen for the NA36 Collaboration, Nucl. Phys. A 566, 217c (1994).

[32] D. Di Bari for the WA85 Collaboration, Nucl. Phys. A 590, 307c (1995).

[33] H.C. Fenker et al., Phys. Rev. D 30, 872 (1984).

[34] S. Mikocki et al., Phys. Rev. D 34, 42 (1986). 
[35] S.F. Biagi et al., Z. Phys. C - Particles and Fields 9, 305 (1981).

[36] S.F. Biagi et al., Z. Phys. C - Particles and Fields 34, 187 (1987).

[37] W. Brückner et al., Nucl. Instrum. Methods A378, 451 (1996).

[38] W.Beusch, CERN-SPSC/77-70, CERN, Geneva, Switzerland (1977)

[39] W. Beusch et al., Nucl. Instrum. Methods A323, 373 (1992);

U. Müller et al., Nucl. Instrum. Methods A371, 27 (1996).

[40] W. Brückner et al., Nucl. Instrum. Methods A313, 345 (1992).

[41] C.V.Scheel, Dissertation,University Amsterdam, 1994.

[42] H.W.Atherton et al., CERN 80-07, CERN, Geneva, Switzerland (1980)

[43] B. Andersson et al., Z.Phys. C57, 485-494 (1993);

H. Pi, Comput. Phys. Commun. 71,173-192 (1992).

[44] GEANT 3.21 CERN Program Library W5103, CERN 1993

[45] R. Blankenbecler and S.J. Brodsky, Phys. Rev. D 10, 2973 (1974).

[46] M. Banner et al., Phys. Lett. 44B, 537 (1973).

[47] J.W. Cronin et al., Phys. Rev. D 11, 3105 (1975).

[48] W.M. Geist, Nucl. Phys. A525, 149c (1991).

[49] D.S. Barton et al., Phys. Rev. D 27, 2580 (1983); within the range of $0 \leq x_{F} \leq 1$, this parametrization is consistent with a more recent compilation given in ref. [48]. 


\begin{tabular}{|c|c|c|c|c|c|c|}
\hline Beam & \multicolumn{2}{|c|}{ Neutrons } & \multicolumn{2}{c|}{$\pi^{-}$} & \multicolumn{2}{c|}{$\Sigma^{-}$} \\
\hline$x_{F}$ & Copper & Carbon & Copper & Carbon & Copper & Carbon \\
\hline $0.0-0.1$ & $49.33 \pm 6.50$ & $10.26 \pm 1.40$ & $23.59 \pm 2.88$ & $3.61 \pm 0.41$ & $29.82 \pm 1.11$ & $9.30 \pm 0.33$ \\
$0.1-0.2$ & $26.22 \pm 1.81$ & $7.38 \pm 0.55$ & $7.39 \pm 0.57$ & $2.41 \pm 0.20$ & $30.01 \pm 0.66$ & $8.39 \pm 0.20$ \\
$0.2-0.3$ & $18.57 \pm 1.33$ & $4.02 \pm 0.31$ & $4.81 \pm 0.43$ & $1.06 \pm 0.11$ & $28.80 \pm 0.65$ & $7.96 \pm 0.18$ \\
$0.3-0.4$ & $7.32 \pm 0.70$ & $1.90 \pm 0.20$ & $3.17 \pm 0.37$ & $0.74 \pm 0.09$ & $26.46 \pm 0.64$ & $8.47 \pm 0.21$ \\
$0.4-0.5$ & $2.49 \pm 0.40$ & $0.67 \pm 0.12$ & $1.46 \pm 0.26$ & $0.60 \pm 0.10$ & $19.74 \pm 0.53$ & $6.71 \pm 0.19$ \\
$0.5-0.6$ & - & $0.26 \pm 0.10$ & $0.52 \pm 0.19$ & $0.29 \pm 0.08$ & $12.58 \pm 0.40$ & $5.16 \pm 0.17$ \\
$0.6-0.7$ & - & - & - & - & $8.32 \pm 0.37$ & $3.40 \pm 0.14$ \\
$0.7-0.8$ & - & - & - & - & $3.93 \pm 0.29$ & $1.61 \pm 0.11$ \\
$0.8-0.9$ & - & - & - & - & $0.44 \pm 0.26$ & $0.45 \pm 0.10$ \\
$0.9-1.0$ & - & - & - & - & - & - \\
\hline
\end{tabular}

Table 1: Differential cross section of $\Xi^{-}$production as a function of $x_{F}$ in mb.

\begin{tabular}{|c|c|c|c|c|c|c|}
\hline Beam & \multicolumn{2}{|c|}{ Neutrons } & \multicolumn{2}{|c|}{$\pi^{-}$} & \multicolumn{2}{c|}{$\Sigma^{-}$} \\
\hline$p_{t}^{2}$ & Copper & Carbon & Copper & Carbon & Copper & Carbon \\
\hline $0.0-0.2$ & $20.53 \pm 1.07$ & $4.43 \pm 0.25$ & $5.96 \pm 0.39$ & $1.54 \pm 0.10$ & $24.84 \pm 0.40$ & $8.45 \pm 0.13$ \\
$0.2-0.4$ & $11.15 \pm 0.82$ & $3.42 \pm 0.25$ & $4.21 \pm 0.33$ & $0.90 \pm 0.08$ & $15.37 \pm 0.34$ & $5.44 \pm 0.12$ \\
$0.4-0.6$ & $8.76 \pm 0.78$ & $1.81 \pm 0.18$ & $4.29 \pm 0.46$ & $0.74 \pm 0.09$ & $11.14 \pm 0.32$ & $3.30 \pm 0.10$ \\
$0.6-0.8$ & $4.92 \pm 0.67$ & $0.91 \pm 0.14$ & $2.21 \pm 0.32$ & $0.51 \pm 0.08$ & $7.35 \pm 0.27$ & $2.56 \pm 0.10$ \\
$0.8-1.0$ & $2.37 \pm 0.52$ & $0.81 \pm 0.18$ & $1.34 \pm 0.28$ & $0.26 \pm 0.07$ & $5.52 \pm 0.25$ & $1.73 \pm 0.08$ \\
$1.0-1.2$ & $2.34 \pm 0.59$ & $0.29 \pm 0.11$ & $1.29 \pm 0.36$ & $0.18 \pm 0.08$ & $4.29 \pm 0.25$ & $1.29 \pm 0.08$ \\
$1.2-1.4$ & $1.44 \pm 0.78$ & $0.00 \pm 0.00$ & $1.17 \pm 0.60$ & $0.21 \pm 0.13$ & $3.78 \pm 0.28$ & $1.03 \pm 0.08$ \\
$1.4-1.6$ & - & - & - & - & $2.84 \pm 0.24$ & $0.78 \pm 0.07$ \\
$1.6-1.8$ & - & - & - & - & $2.58 \pm 0.27$ & $0.61 \pm 0.07$ \\
$1.8-2.0$ & - & - & - & - & $2.22 \pm 0.29$ & $0.59 \pm 0.08$ \\
$2.0-2.2$ & - & - & - & - & $1.97 \pm 0.32$ & $0.71 \pm 0.15$ \\
$2.2-2.4$ & - & - & - & - & $1.67 \pm 0.34$ & $0.49 \pm 0.10$ \\
\hline
\end{tabular}

Table 2: Differential cross section of $\Xi^{-}$production as a function of $p_{t}^{2}$ in $\mathrm{mb} /(\mathrm{GeV} / c)^{-2}$. 


\begin{tabular}{|l|l|c|c|l|c|c|}
\hline $\begin{array}{l}\text { Beam } \\
\text { particle }\end{array}$ & Target & \# events & $\begin{array}{l}\text { average total } \\
\text { efficiency }\end{array}$ & $\begin{array}{l}\text { cross section per } \\
\text { nucleus }(m b)\end{array}$ & $\mathrm{n}$ & $\begin{array}{c}b \\
(\mathrm{GeV} / c)^{-2}\end{array}$ \\
\hline$\Sigma^{-}$ & Copper & $90160 \pm 336$ & 0.029 & $18.31 \pm 0.09$ & $1.97 \pm 0.04$ & $1.90 \pm 0.04$ \\
& Carbon & $87528 \pm 331$ & 0.029 & $5.15 \pm 0.03$ & $2.08 \pm 0.04$ & $2.00 \pm 0.04$ \\
\hline Neutrons & Copper & $1847 \pm 47$ & 0.034 & $10.6 \pm 0.1$ & $4.8 \pm 0.3$ & $2.3 \pm 0.2$ \\
& Carbon & $1627 \pm 44$ & 0.034 & $2.5 \pm 0.1$ & $5.0 \pm 0.3$ & $2.4 \pm 0.2$ \\
\hline$\pi^{-}$ & Copper & $1683 \pm 48$ & 0.043 & $4.1 \pm 0.1$ & $4.1 \pm 0.3$ & $1.6 \pm 0.1$ \\
& Carbon & $1448 \pm 44$ & 0.043 & $0.9 \pm 0.1$ & $3.8 \pm 0.3$ & $1.9 \pm 0.1$ \\
\hline
\end{tabular}

Table 3: Number of reconstructed events, average efficiency, total inclusive $\Xi^{-}$production cross sections and parameters of fits using a function of the form $d^{2} \sigma / d p_{t}^{2} d x_{F}=C\left(1-x_{F}\right)^{n} e x p\left(-b p_{t}^{2}\right)$ for the different beam particles and targets. Only statistical errors are given.

\begin{tabular}{|c|c|c|c|c|c|}
\hline & \multicolumn{5}{|c|}{$E \cdot d^{3} \sigma / d p^{3}\left[\mathrm{mb} \cdot \mathrm{c}^{3} \cdot \mathrm{GeV}^{-2}\right]$} \\
$x_{F}$ & $0.0<p_{t}^{2}<0.4$ & $0.4<p_{t}^{2}<0.8$ & $0.8<p_{t}^{2}<1.2$ & $1.2<p_{t}^{2}<1.6$ & $1.6<p_{t}^{2}<2.0$ \\
\hline $0.0-0.2$ & $30.2 \pm 0.6$ & $15.2 \pm 0.7$ & $10.8 \pm 0.8$ & $10.2 \pm 1.5$ & $8.9 \pm 2.3$ \\
$0.2-0.4$ & $88.3 \pm 1.7$ & $42.7 \pm 1.7$ & $23.6 \pm 1.6$ & $15.7 \pm 1.7$ & $19.4 \pm 4.6$ \\
$0.4-0.6$ & $95.8 \pm 2.3$ & $46.6 \pm 2.0$ & $23.2 \pm 1.6$ & $17.7 \pm 2.0$ & $11.2 \pm 2.2$ \\
$0.6-0.8$ & $67.5 \pm 2.3$ & $34.6 \pm 1.9$ & $21.7 \pm 2.0$ & $17.0 \pm 2.6$ & $9.0 \pm 2.7$ \\
$0.8-1.0$ & $38.8 \pm 2.6$ & $12.9 \pm 1.4$ & $9.7 \pm 2.5$ & - & - \\
\hline
\end{tabular}

Table 4: Invariant cross section of $\Xi^{-}$production by $\Sigma^{-}$on copper as a function of $x_{F}$ and $p_{t}^{2}$. 


\begin{tabular}{|c|c|c|c|c|c|}
\hline & \multicolumn{4}{|c|}{$E \cdot d^{3} \sigma / d p^{3}\left[\mathrm{mb} \cdot \mathrm{c}^{3} \cdot \mathrm{GeV}^{-2}\right]$} & \\
$x_{F}$ & $0.0<p_{t}^{2}<0.4$ & $0.4<p_{t}^{2}<0.8$ & $0.8<p_{t}^{2}<1.2$ & $1.2<p_{t}^{2}<1.6$ & $1.6<p_{t}^{2}<2.0$ \\
\hline $0.0-0.2$ & $8.9 \pm 0.2$ & $4.1 \pm 0.2$ & $2.8 \pm 0.2$ & $2.2 \pm 0.3$ & $2.3 \pm 0.8$ \\
$0.2-0.4$ & $27.5 \pm 0.6$ & $12.6 \pm 0.5$ & $6.2 \pm 0.4$ & $3.8 \pm 0.4$ & $3.9 \pm 0.9$ \\
$0.4-0.6$ & $35.8 \pm 0.9$ & $15.1 \pm 0.7$ & $8.8 \pm 0.7$ & $6.0 \pm 0.8$ & $3.3 \pm 0.6$ \\
$0.6-0.8$ & $30.2 \pm 1.0$ & $10.7 \pm 0.6$ & $6.9 \pm 0.6$ & $5.9 \pm 1.0$ & $2.7 \pm 1.0$ \\
$0.8-1.0$ & $15.9 \pm 1.0$ & $8.4 \pm 0.9$ & $3.2 \pm 0.7$ & - & - \\
\hline
\end{tabular}

Table 5: Invariant cross section of $\Xi^{-}$production by $\Sigma^{-}$on carbon as a function of $x_{F}$ and $p_{t}^{2}$.

\begin{tabular}{|l|c|c|}
\hline $\begin{array}{l}\text { Beam } \\
\text { particle }\end{array}$ & $\alpha$ & $\begin{array}{c}\text { cross section per } \\
\text { nucleon, } m b\left(x_{F}>0\right)\end{array}$ \\
\hline 1. $\Sigma^{-}$ & $0.681 \pm 0.001$ & $1.06 \pm 0.01$ \\
2. Neutrons & $0.880 \pm 0.04$ & $0.24 \pm 0.10$ \\
3. $\pi^{-}$ & $0.920 \pm 0.03$ & $0.11 \pm 0.09$ \\
\hline
\end{tabular}

Table 6: $A$ dependence of the inclusive cross section of the $\Xi^{-}$production. 
WA89

Hyperon beam

1993 layout
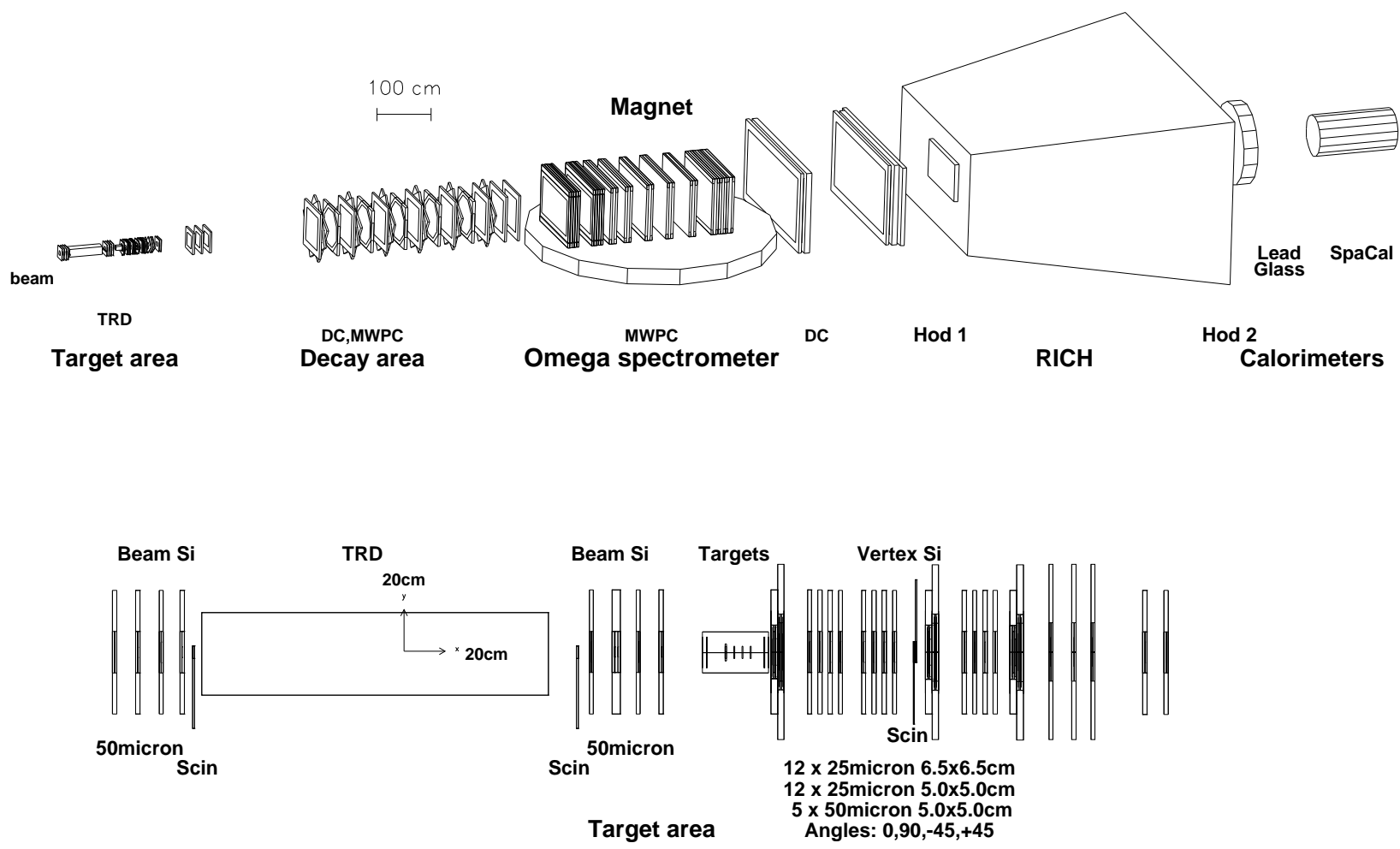

Figure 1: Setup of the WA89 experiment in the 1993 run. The lower part shows an expanded view of the target area. 


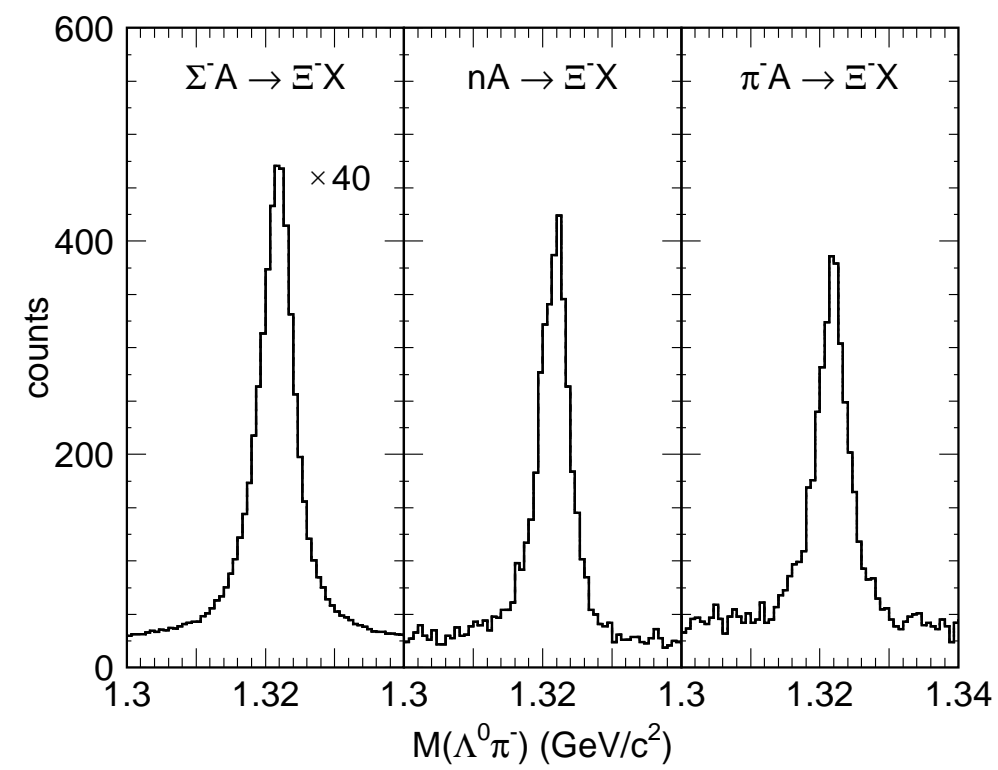

Figure 2: Invariant mass distributions of $\Lambda^{0}-\pi^{-}$pairs produced in $\Sigma^{-}$(left), neutron (centre), and $\pi^{-}$(right) interactions. The data for both targets have been added. 

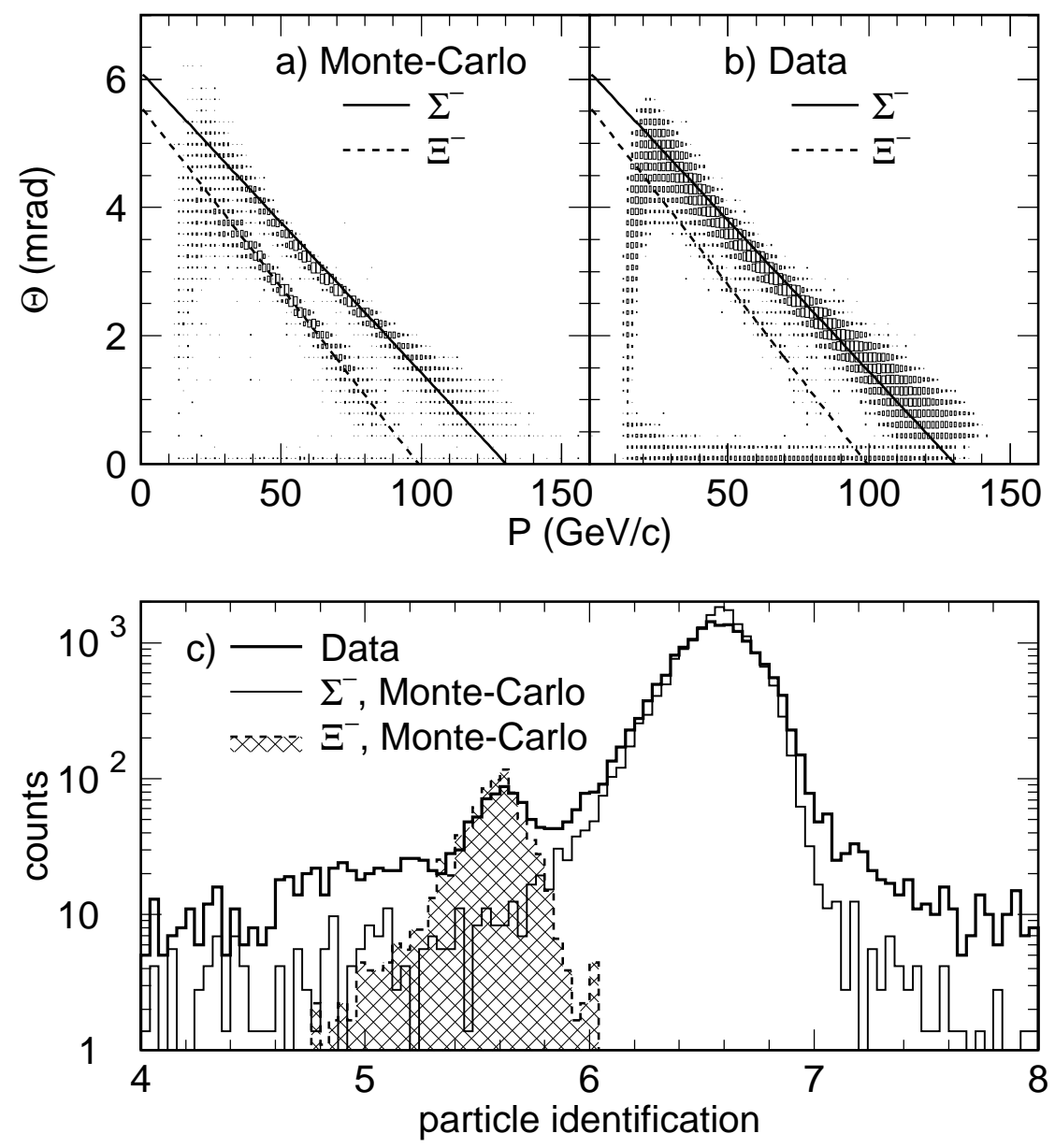

Figure 3: Correlation plots between momentum and decay angle between incoming $\Sigma^{-}$and $\Xi^{-}$and outgoing $\pi^{-}$for a) Monte Carlo and b) Data. The projections of these distributions along the two indicated lines is shown in part c). 


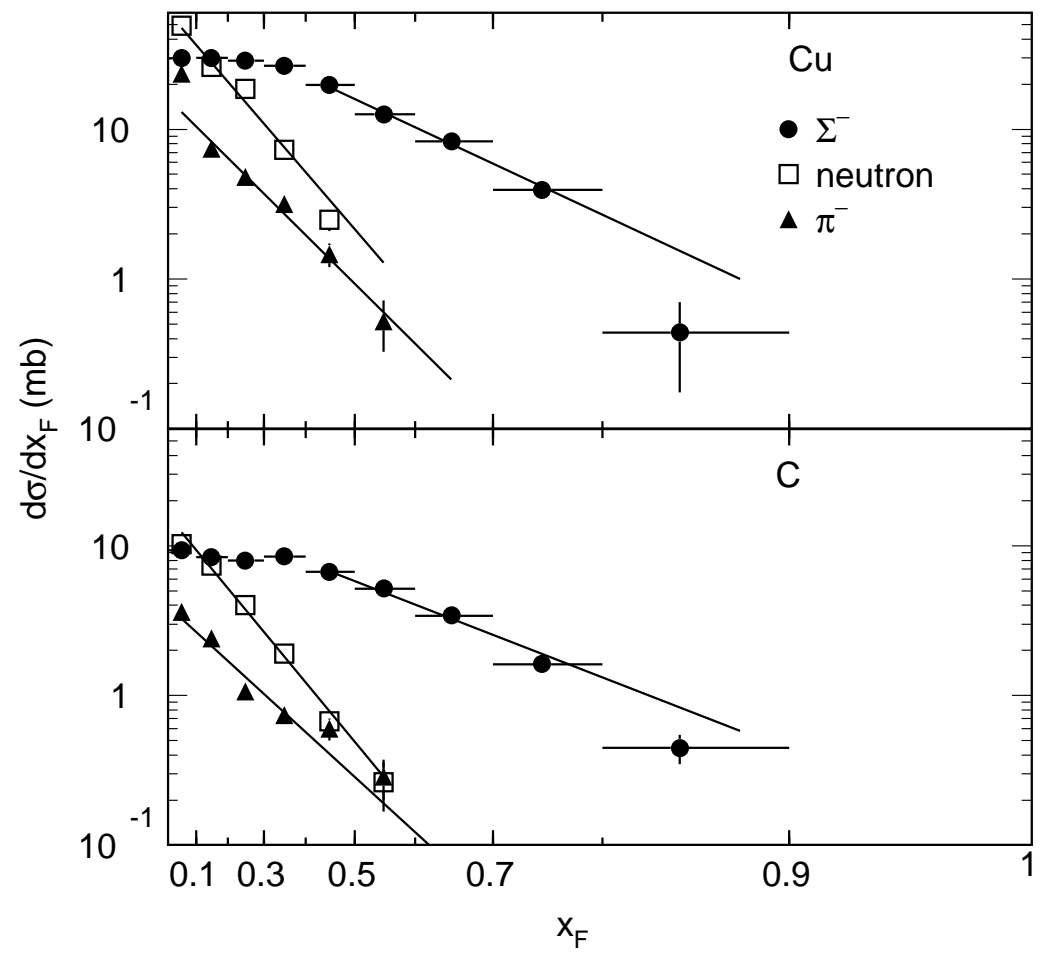

Figure 4: The differential cross section $d \sigma / d x_{F}$ as a function of $x_{F}$ integrated over $p_{t}^{2}$ for neutron, $\pi^{-}$and $\Sigma^{-}$interactions with copper (top part) and carbon (bottom part). The lines represent fits proportional to $\left(1-x_{F}\right)^{n}$ with the parameters $\mathrm{n}$ as given in table 3 . 


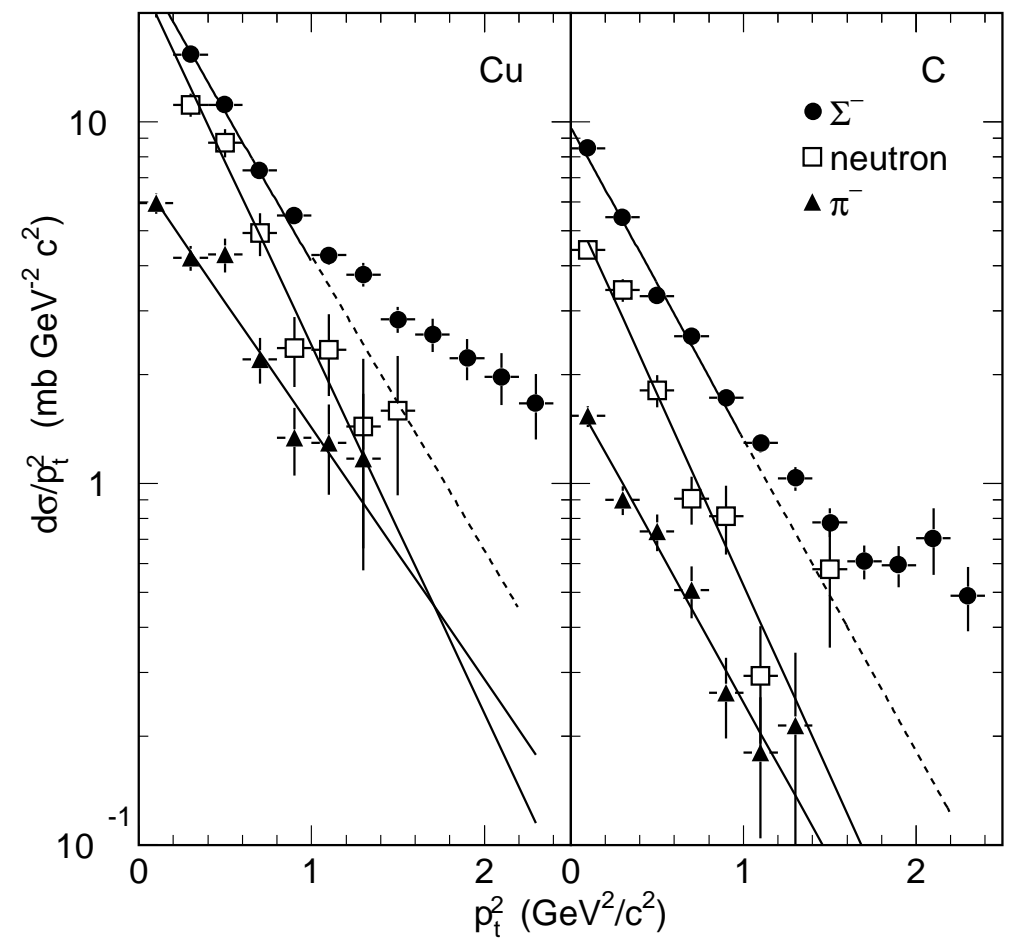

Figure 5: The differential cross section as a function of $p_{t}^{2}$ integrated over $x_{F}$ for neutron, $\pi^{-}$and $\Sigma^{-}$interaction with copper (left part) and carbon (right part). The lines represent fits proportional to $\exp \left(-b p_{t}^{2}\right)$; the parameters $\mathrm{b}$ are listed in table 3 . 


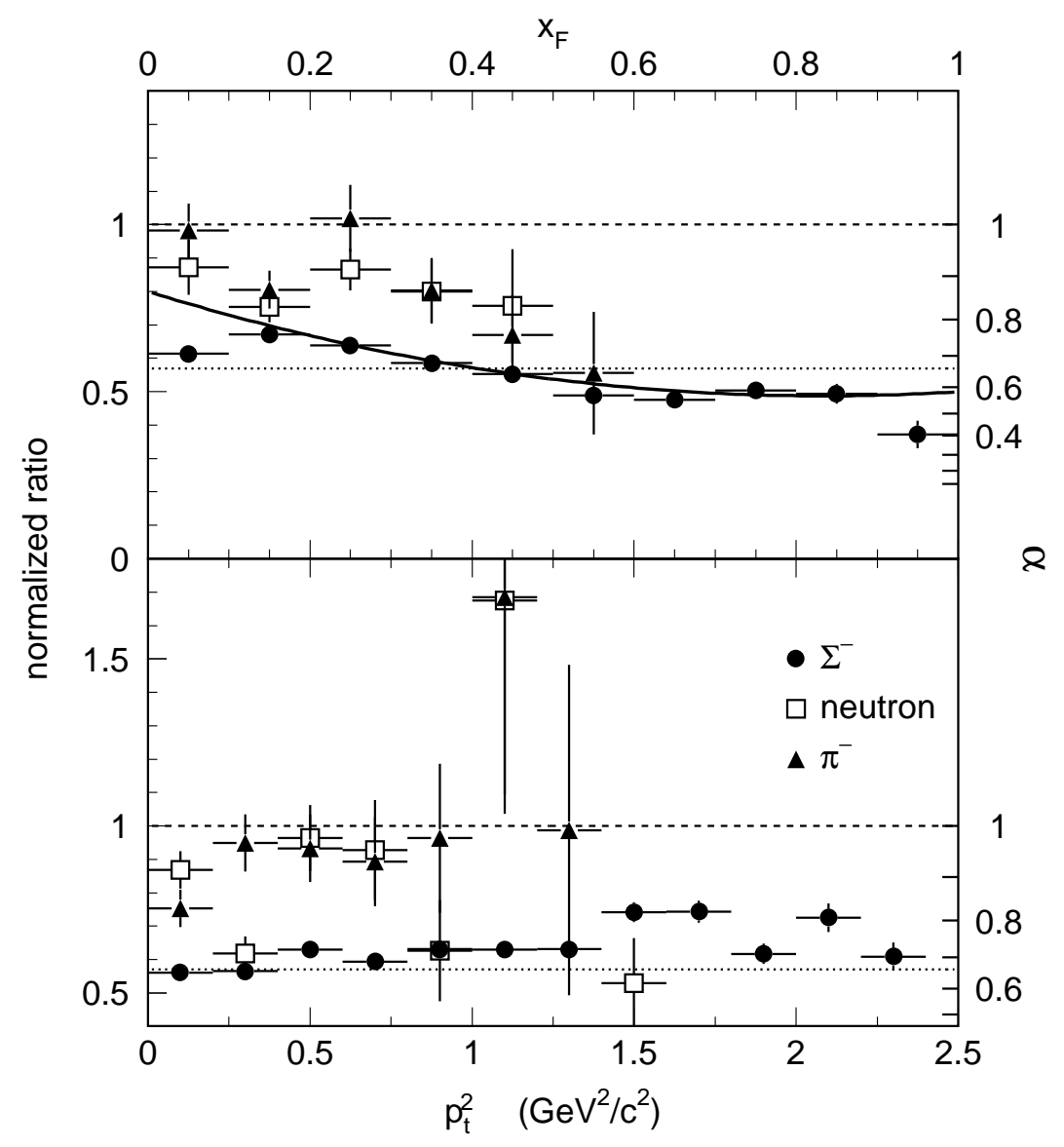

Figure 6: Normalized ratio $R$ (see Eq. 4) of the $\Xi^{-}$cross section measured in reactions with carbon and copper nuclei as a function of $x_{F}$ (top part) and $p_{t}^{2}$ (bottom part) for neutron, $\pi^{-}$and $\Sigma^{-}$ induced reactions. The right scale indicates the exponent $\alpha$ in case of an $A^{\alpha}$ dependence. The solid line in the upper part marks a polynomial fit to a compilation of target attenuation factors given in ref. [48]. 


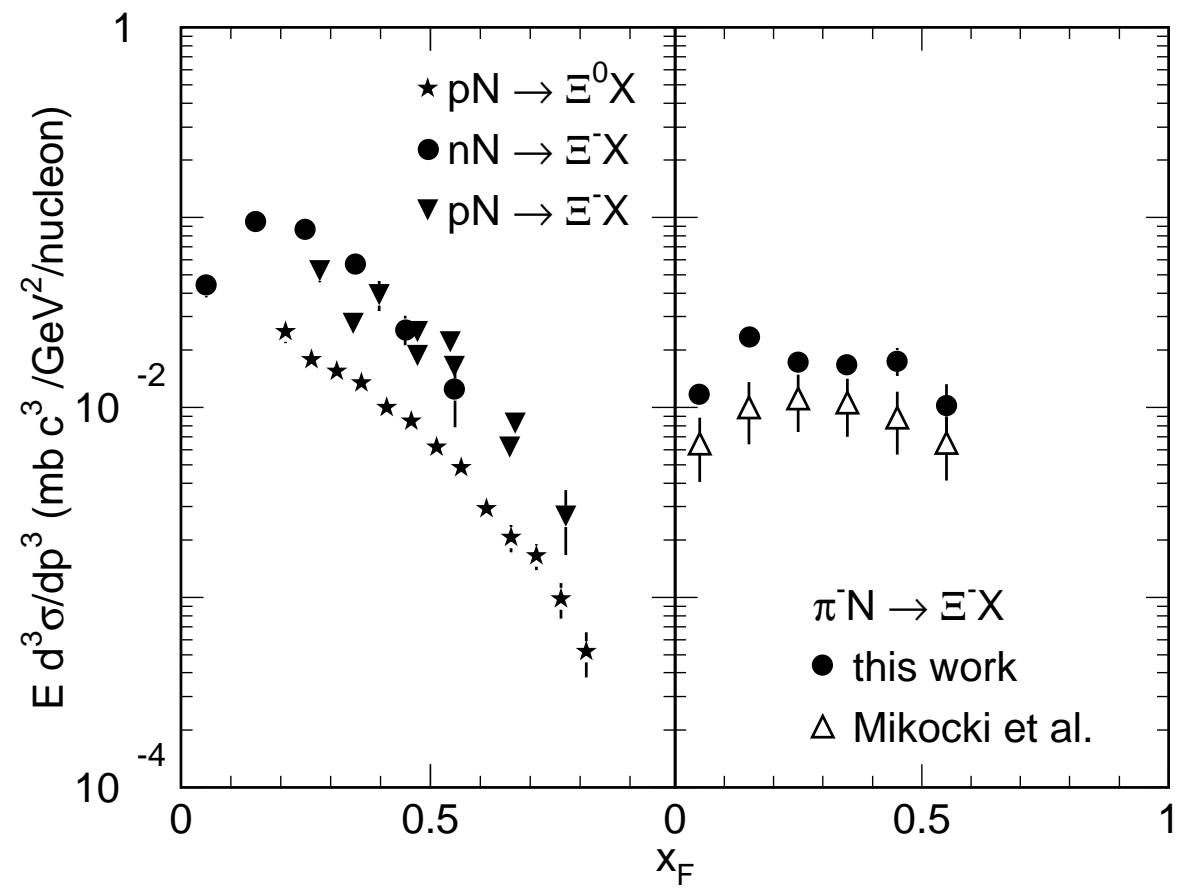

Figure 7: Left part: Invariant cross sections for inclusive $\Xi^{-}$and $\Xi^{0}$ production at $p_{t}^{2}<0.2 \mathrm{GeV}^{2} / c^{2}$ by protons and neutrons: $\mathrm{n} A \rightarrow \Xi^{-} X$ at $345 \mathrm{GeV} / c$ this measurement (filled circles); $\mathrm{p} A \rightarrow \Xi^{-} X$ at $200 \mathrm{GeV} / c$ and $400 \mathrm{GeV} / c[22,26]$ (triangles); $\mathrm{p} A \rightarrow \Xi^{0} X$ at $400 \mathrm{GeV} / c[27]$ (stars). The right part compares the invariant inclusive $\Xi^{-}$production cross sections at $p_{t}^{2}<0.2 \mathrm{GeV}^{2} / c^{2}$ by pions observed in the present experiment at $345 \mathrm{GeV} / c$ (filled circles), to previous data taken at 200 $\mathrm{GeV} / c$ (open triangles) [34]. 


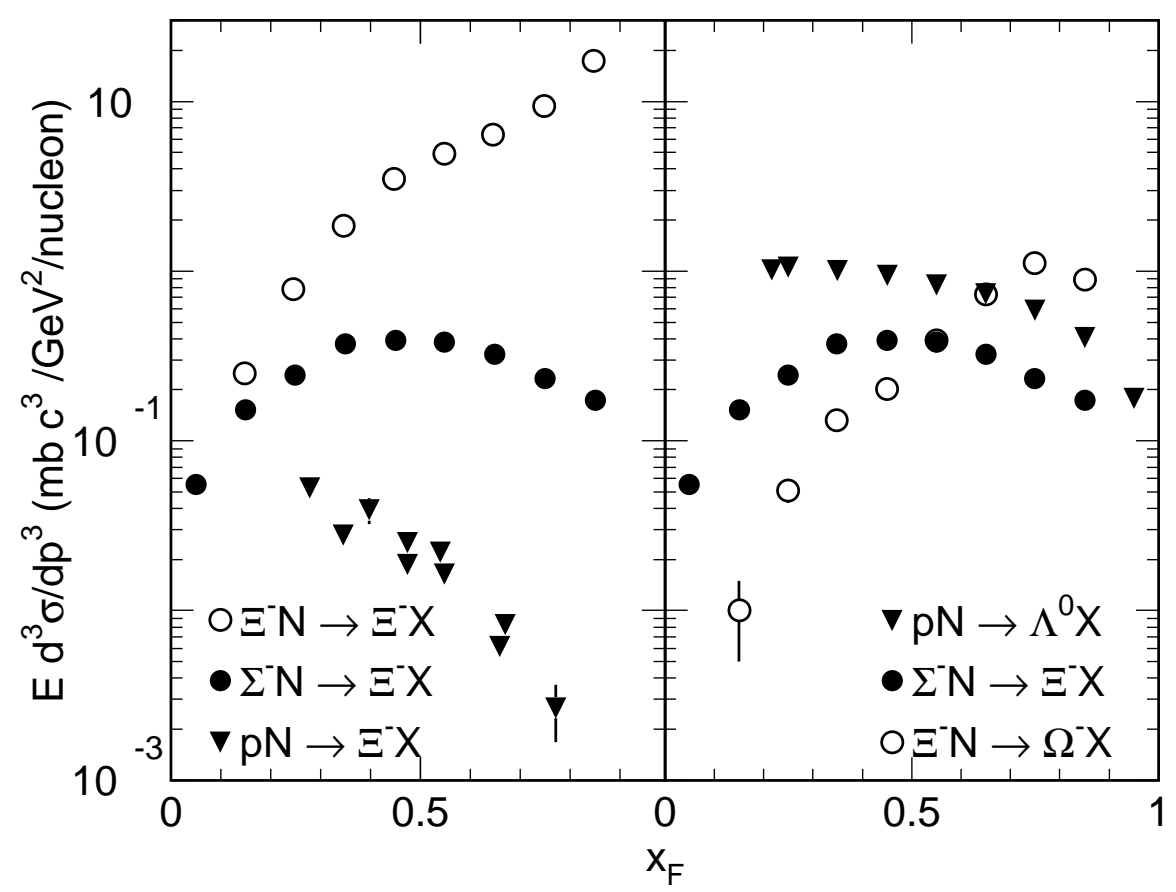

Figure 8: Invariant cross sections per nucleon for baryons containing strange quarks. The left part displays the $\Xi^{-}$cross section at $p_{t}^{2}<0.2 \mathrm{GeV}^{2} / c^{2}$ in reactions with $\Delta \mathrm{S}=0$ (open circles), $\Delta \mathrm{S}=1$ (filled circles), and $\Delta \mathrm{S}=2$ (triangles) between beam and observed particle. The invariant inclusive particle production cross section at $p_{t}^{2}<0.2 \mathrm{GeV}^{2} / c^{2}$ in reactions with $\Delta S=1$ between projectiles and observed final particle for different projectiles is shown on the right hand side. The data are from $\Xi^{-} A \rightarrow \Xi^{-} X$ and $\Xi^{-} A \rightarrow \Omega^{-} X$ at $116 \mathrm{GeV} / c$ [36], $\Sigma^{-} A \rightarrow \Xi^{-} X$ at $345 \mathrm{GeV} / c$ (this measurement), $\mathrm{p} A \rightarrow \Xi^{-} X$ at $200 \mathrm{GeV} / c$ and at $400 \mathrm{GeV} / c[22,26]$, and $\mathrm{p} A \rightarrow \Lambda^{0} X$ at 300 $\mathrm{GeV} / c[21]$. 